\title{
A Filament Model of MHD Turbulence
}

\author{
V. Petviashvili ${ }^{a}$
}

Turbulence of ordinary fluid is recognized as chaotic motion with almost no linear features. It is well described in wavenumber space by Kolmogorov's phenomenological theory in wave number k-space: The source of energy should exist in the region of small wavenumbers. Then isotropic energy flux is generated in $\mathbf{k}$-space directed toward a larger $\mathbf{k}$-region where the energy is absorbed by viscosity. The main characteristics of energy spectrum of Kolmogorov turbulence is universal and in good agreement with observations.

When fluid is electrically conducting, the magnetic field is generated by turbulence. The resulting MHD turbulence is more complicated as it contains more parameters. Kolmogorov's method was applied to it by many authors. Kraichnan [1] noticed the feature of MHD turbulence which makes it locally anisotropic: the small scale perturbations propagate like waves due to large scale magnetic field with Alfvén velocity

$$
\mathbf{c}_{A}= \pm(4 \pi \rho)^{-1 / 2} \mathbf{B}
$$

It is important that (1) has two directions. In usual hydrodynamics small scale perturbations are flown along large scale perturbations of velocity as flows, not as waves (i.e. with substance).

Kraichnan's spectrum is not in good agreement with observations of MHD turbulence spectrum in solar wind: It ascribes to large scale perturbations less amplitudes than are observed. Besides this, observations in the solar atmosphere suggest that MHD turbulence contains filament-like structures, while in Kraichnan's and Kolmogorov's turbulence as structures are supposed to exist.

\footnotetext{
${ }^{a)}$ Deceased July 28, 1993. Visitor at the Institute for Fusion Studies, The University of Texas at Austin, Austin, TX 78712
} 
Some time ago, the equations of current and vorticity filaments in MHD approximation were derived . Here we suppose that MHD turbulence consists of chaotic set of such filaments (Alfvén filaments) and no energy flux exists in $\mathbf{k}$-space. So filaments are in thermal equilibrium like long molecules in organic chemistry. This model can be justified in a hot enough plasma, where Larmor radius is less than free path of particles. In this case dispersive effects influence much stronger than dissipation and this can stop energy flux. Take for comparison ion-acoustic turbulence: dispersion even reverses flux towards the large scale region in it. In ordinary hydrodynamics vortex filaments are subject to viscous damping and this makes Kolmogorov's turbulence structureless. Nevertheless some traces of filaments were observed in ordinary turbulence as well.

The existence of a set of Alfvén filaments in thermal equilibrium can be responsible for the presence of large scale magnetic structures in primordial plasma. This in turn may result in strongly inhomogeneous distribution of matter in the universe during expansion, due to freezing of plasma in nonuniform magnetic field.

Alfvén waves are closely connected with motion of a magnetized plasma, because of their incompressibility and low velocity across magnetic field. This gives a notion that the analog of vortex filament in nonconductive fluid may be Alfvén vortex filament, plasma velocity and magnetic field circulating around it and connected by Alfvén relation. To show this we start from MHD equations written in symmetric form, suggested by Elsasser [2]

$$
\begin{array}{ll}
\left(\partial_{t}+\mathbf{w} \cdot \nabla\right) \mathbf{u}=-\nabla p & \operatorname{div} \mathbf{w}=\operatorname{div} \mathbf{u}=0 . \\
\left(\partial_{t}+\mathbf{u} \cdot \nabla\right) \mathbf{w}=-\nabla p & \nabla^{2} p=-\operatorname{div}(\mathbf{w} \cdot \nabla) \mathbf{u}
\end{array}
$$

where

$$
\mathbf{u}=\mathbf{v}+\mathbf{c}_{A} ; \quad \mathbf{w}=\mathbf{v}-\mathbf{c}_{A} ; \quad \mathbf{c}_{A}=(4 \pi \rho)^{-1 / 2} \mathbf{B}
$$

$\mathbf{v}$ is plasma velocity and $\mathbf{B}$ is magnetic field. Derivatives of density $\rho$ are neglected. It follows 
from (2) and (3) that if plasma flows with local Alfvén velocity $\pm \mathbf{c}_{A}$ (i.e. $\mathbf{w}=0$ or $\mathbf{u}=0$ ) then interaction disappears and we have stationary state $\left(\partial_{t}=0, \nabla p=0\right)$.

Let us consider trajectories along which weak disturbances propagate (i.e. characteristics). There are two sets of them which we call upstream and downstream ones. The following equations follow from (2) and (3) for them:

$$
\partial_{t} \mathbf{r}=\mathbf{w}(\mathbf{z}, t) ; \quad \partial_{t} \mathbf{r}=\mathbf{u}(\mathbf{r}, t) .
$$

We see that if $\mathbf{w}=0$, there is no interaction between perturbations of $\mathbf{u}$, and $\mathbf{w}$ if $\mathbf{u}=0$. We suppose the existence of filament-like singularities in the form:

$$
\begin{aligned}
& \operatorname{curl} \mathbf{u}=4 \pi \sum_{i} \alpha_{i} \oint \delta_{3}\left(\boldsymbol{\tau}-\boldsymbol{\tau}_{i}\right) \boldsymbol{\tau}_{i}^{\prime} d s_{i} \\
& \operatorname{curl} \mathbf{w}=4 \pi \sum_{j} \beta_{j} \int \delta_{3}\left(\tau-\tau_{j}\right) \boldsymbol{\tau}_{j}^{\prime} d s_{j} \\
& \mathbf{u}=\operatorname{curl} \sum_{i} \alpha_{i} \mathbf{A}_{i} \quad \mathbf{w}=\operatorname{curl} \sum_{j} \beta_{j} \mathbf{A}_{j} \\
& \mathbf{A}_{i}=-\int\left|r-r_{i}\right|^{-1} \mathbf{r}_{i}^{\prime} d s_{i} ; \quad \mathbf{r}^{\prime} \equiv \partial_{s} \mathbf{r} .
\end{aligned}
$$

Here $\mathbf{r}_{i}\left(t, s_{i}\right)$ is coordinate of filament, dependent on time $t$ and curve parameter $s_{i}$. Integration on $s_{i}$ is carried out along a curve labeled by $i . \alpha_{i}, \beta_{j}$ are positive constant charges of filaments of different types. The number of filaments may be infinite. Filaments may be closed curves. Then $\mathbf{r}$ is a periodic function of $s$. Substituting (5) in (4) and taking $\mathbf{r}$ to be the filament's coordinate, we obtain

$$
\begin{aligned}
\partial_{t} \mathbf{r}_{i} & =\sum_{j} \beta_{j} \oint \mathbf{R}_{i j} d s_{j} \\
\partial_{t} \mathbf{r}_{j} & =\sum_{i} \alpha_{i} \int R_{j i} d s_{i} \\
\mathbf{R}_{i j} & =\left(\mathbf{r}_{i}-\mathbf{r}_{j}\right) \times \mathbf{r}_{j}^{\prime}\left|\mathbf{r}_{i}-\mathbf{r}_{j}\right|^{-3} .
\end{aligned}
$$


This system is invariant relative to translation and rotation in space, so it has corresonding integrals of motion. It also conserves energy in the form:

$$
E=\sum \alpha_{i} \beta_{j} \iint \frac{\mathbf{r}_{i}^{\prime} \cdot \mathbf{r}_{j}^{\prime}}{\left|\mathbf{r}_{i}-\mathbf{r}_{j}\right|} d s_{i} d s_{j}
$$

So we see that MHD equations in the presence of filament turbulence are reduced to a set of integro-differential equations (6) and (7).

The type of filament is determined by sign of cross helicity $h_{i}=\mathbf{v}_{i} \cdot \mathbf{B}_{i}$ where $\mathbf{B}_{i}$ is a magnetic field created by current in the $i$-th filament, $\mathbf{v}_{i}= \pm(4 \pi \rho)^{-1 / 2} \mathbf{B}_{i}$ is corresponding velocity of plasma circulation around the filament. So we see that filaments interact hydrodynamically and magnetically. The filaments with the same sign of $h$ do not interact because magnetic interaction is cancelled by hydrodynamic ones. If helicities have opposite sign, the interaction of a different nature are summed.

Note that (9) is diverging only logarithmically when $\mathbf{r}_{i}$ tends to coincide with $\mathbf{r}_{j}$. Because of this an evaluation of $E$ is possible.

$$
E \sim \sum_{i j} \frac{\mathbf{m}_{i} \cdot \mathbf{m}_{j}}{\left(R_{i j}^{2}+\rho_{i} \rho_{j}\right)^{3 / 2}} .
$$

Here $\mathbf{m}_{i}$ is effective moment directed perpendicular to $i$-th loop. $R_{i j}$ is distance between centers of loop, $\rho_{i}$ is effective radius of loop.

Let us evaluate the power index in spectrum of turbulence consisting of filament singularities: Fourier transformation gives:

$$
\begin{aligned}
& \mathbf{B}(\boldsymbol{\tau}, t)=\int \mathbf{B}_{\mathbf{k}}(t) e^{i \mathbf{k} \boldsymbol{\tau}} d^{3} \mathbf{k} \\
& \operatorname{curl} \mathbf{B}=\sum \alpha_{i} \int \delta_{3}\left(\boldsymbol{\tau}-\boldsymbol{\tau}_{i}\right) \boldsymbol{\tau}_{i}^{\prime} d s=i \int\left(\mathbf{k} \times \mathbf{B}_{\mathbf{k}}\right) e^{i \mathbf{k} \boldsymbol{\tau}} d^{3} k \\
& \delta_{3}\left(\boldsymbol{\tau}-\boldsymbol{\tau}_{i}\right)=\int e^{i \mathbf{k}\left(\boldsymbol{\tau}-\boldsymbol{\tau}_{i}\right)} d^{3} \mathbf{k} \\
& \int \delta_{3}\left(\boldsymbol{\tau}-\boldsymbol{\tau}_{i}\right) \boldsymbol{\tau}_{i}^{\prime} d s_{i} \sim \int e^{i \mathbf{k} \boldsymbol{\tau}} \frac{1}{i k} d^{3} \mathbf{k} .
\end{aligned}
$$


Comparing (11) and (12) we obtain

$$
\mathbf{k} \times \mathbf{B}_{k} \sim \frac{1}{k} \quad B_{k} \sim \frac{1}{k^{2}}
$$

Energy $W$ is proportional to

$$
W=\int B^{2} d^{3} \mathbf{r}=\int\left|B_{k}\right|^{2} d^{3} \mathbf{k}=\int W_{k} d k .
$$

From (13) and (14) it follows:

$$
W_{k} \sim k^{-2}
$$

as compared to Kraichnan's where $W_{k} \sim k^{-3 / 2}$.

Note:

Vladimir Petviashvili, a visitor at the Institute for Fusion Studies, left this manuscript with Toshi Tajima, who collaborated with Vladimir and has followed his program and idea. Though this note never became a paper while he was alive, the program that followed his idea has been carried out by his son, Nikolai, Tajima's student, Rodney Kinney, and T. Tajima. R. Kinney wrote a Ph.D. Thesis on this subject in 1992 and published a couple of papers on the subject:

R. Kinney, T. Tajima, N. Petviashvili, and J.C. McWilliams, Phys. Rev. Lett. 71, 1712 (1993).

R. Kinney, T. Tajima, J.C. McWilliams, and N. Petviashvili, Phys. Plasmas 1, 260 (1994).

It also heavily influenced the work of

S.R. Oliveira and T. Tjima, Phys. Rev. E 51, 4287 (1995).

V. Petviashvili's legacy and soul live in these papers, showing his foresight and deep physics insight. 


\section{References}

1. Kraichnan, R.H., Phys. Fluids 8, 1385-1387 (1965).

2. Elsasser, W.M. Phys. Rev. 79, 183 (1950). 